\section{(O) OPEN ACCESS}

\title{
The Massachusetts General Hospital acute stroke imaging algorithm: an experience and evidence based approach
}

\author{
Ramon Gilberto González, William A Copen, Pamela W Schaefer, Michael H Lev, \\ Stuart R Pomerantz, Otto Rapalino, John W Chen, George J Hunter, Javier M Romero, \\ Bradley R Buchbinder, Mykol Larvie, Joshua Adam Hirsch, Rajiv Gupta
}

\begin{abstract}
- Additional material is published online only. To view please visit the journal online (http://dx.doi.org/10.1136/ neurintsurg-2013-010715)

Neuroradiology Division, Massachusetts General Hospital, Harvard Medical School, Boston,

Massachusetts, USA
\end{abstract}

Correspondence to Dr R G González, Neuroradiology Division, Massachusetts General Hospital, Harvard Medical School, Boston, MA 02114, USA;rggonzalez@partners.org

Received 21 February 2013 Accepted 21 February 2013 Published Online First

14 March 2013

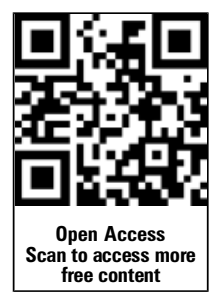

\section{ABSTRACT}

The Massachusetts General Hospital Neuroradiology Division employed an experience and evidence based approach to develop a neuroimaging algorithm to best select patients with severe ischemic strokes caused by anterior circulation occlusions (ACOs) for intravenous tissue plasminogen activator and endovascular treatment. Methods found to be of value included the National Institutes of Health Stroke Scale (NIHSS), non-contrast CT, CT angiography (CTA) and diffusion MRI. Perfusion imaging by CT and MRI were found to be unnecessary for safe and effective triage of patients with severe ACOs. An algorithm was adopted that includes: non-contrast CT to identify hemorrhage and large hypodensity followed by CTA to identify the ACO; diffusion MRI to estimate the core infarct; and NIHSS in conjunction with diffusion data to estimate the clinical penumbra.

\section{INTRODUCTION}

The purpose was to use an experience and evidence based approach to develop the neuroimaging algorithm that best improves outcomes in patients with severe ischemic strokes caused by anterior circulation occlusions (ACOs). Patients with these strokes account for the majority of individual, family, and societal costs due to stroke, and they are treatable with intravenous (IV) tissue plasminogen activator (tPA) or/and intra-arterial therapy (IAT). Critically evaluated was the capability of each specific method to provide reliable information on three key components of stroke physiology: (1) site of arterial occlusion; (2) extent of irreversibly injured tissue ('infarct core'); and (3) the size of the ischemic penumbra (figure 1). Although varying definitions of the ischemic penumbra exist, the penumbra is defined herein as severely hypoperfused brain tissue that may eventually be recruited into the infarct core, if not reperfused quickly enough. ${ }^{1}$

\section{METHODOLOGY}

The critical physiological information in the acute stroke patient with severe symptoms is shown in figure 1 , which is a representation of a patient with a proximal right middle cerebral artery occlusion. Individual neuroradiology and neurology faculty from the Massachusetts General Hospital $(\mathrm{MGH})$ presented the best evidence from the literature and clinical experience on the value of each method. Expert opinions were presented for the National Institutes of Health Stroke Scale (NIHSS), non-contrast CT (NCCT), CT angiography (CTA), CTA source images (CTA-SI), diffusion MRI, CT perfusion (CTP), and MRI perfusion (MRP). Faculty and fellows who did not present but heard the evidence, met to weigh the evidence and make recommendations. Each modality was assessed on two different sets of criteria shown in tables 1 and 2. The basic practical question was: 'Is the modality valuable for patient care and can we obtain it in an acute setting?' In addition to traditional metrics such as sensitivity and specificity, the following factors were factored into the assessments:

- Workflow. Place of the imaging test in the workflow and does it negatively or positively affect the workflow when an acute stroke patient arrives in the emergency department.

- Repeatability. The values measured by the imaging test are not affected by extraneous parameters and repeated invocations of the test would result in the same conclusions.

- Reliability. Measured values reflect the purported physiologic parameter about the patient condition.

- Clinical efficacy. The imaging test improves patient outcomes.

\section{ISCHEMIC STROKE THERAPY}

\section{Intravenous tissue plasminogen activator}

Intravenously administered tPA is a proven effective treatment for ischemic stroke. ${ }^{2}$ The primary indicators for its administration are an elapsed time since onset of $4.5 \mathrm{~h}$ or less and an absence of hemorrhage or large infarct on imaging, usually NCCT.

\section{Intra-arterial therapy}

The target of IAT is a proximal artery occlusion. The focus here is on major ACOs which account for approximately $90 \%$ of all such occlusions. ${ }^{3}$ In patients with blockages of the intracranial internal carotid artery (ICA) or middle cerebral artery stem (M1 segment), two recent studies have demonstrated the critical role of infarct volume in determining long term functional outcome (figure 2). ${ }^{45}$ 


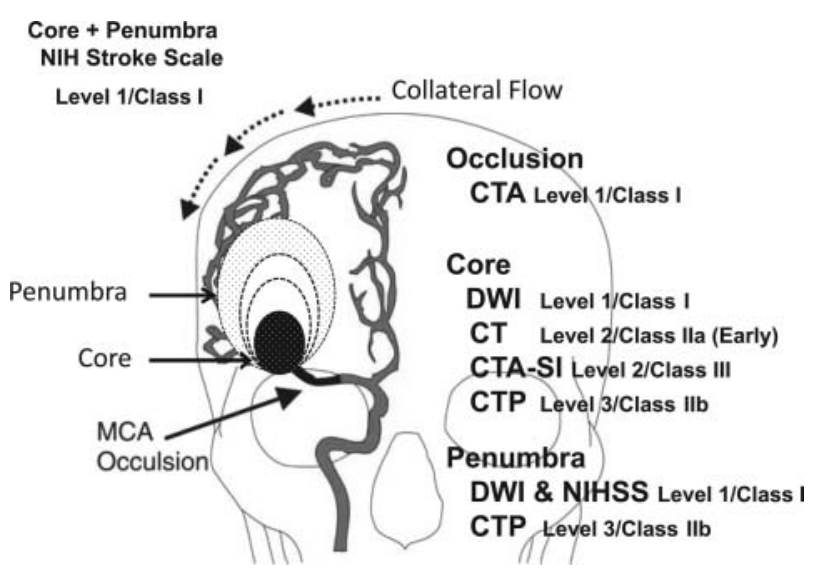

Figure 1 Measurements of physiological components of middle cerebral artery (MCA) occlusion. Depicted is a representation of a right MCA occlusion with a growing infarct core coupled to a shrinking penumbra at a rate determined by collateral flow. The value of each method used to measure each component of stroke physiology is shown, as judged according to criteria stated in the methodology and in tables 1 and 2. CTA, CT angiography; CTA-SI, CT angiography-source images; CTP, CT perfusion; DWI, diffusion weighted imaging; NIHSS, National Institutes of Health Stroke Scale.

When patients have large final infarcts, there is a high likelihood of significant disability or death. It follows then that patients presenting with extensive infarction (ie, >70-100 ml) before treatment will have little chance for a good IAT response. ${ }^{7}$ Moreover, studies have shown that the risk of reperfusion hemorrhage increases with pretreatment infarct size, and is very high ( $15 \%)$ when infarcts are larger than $100 \mathrm{ml}^{8}{ }^{9}$ Therefore, risk outweighs benefit in this subset of patients. Numerous studies have confirmed the importance of

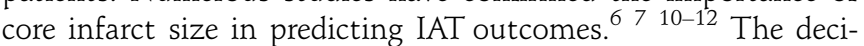
sion to proceed to IAT therefore critically depends on the size of the infarct core at the time of treatment, and the primary role of imaging after identification of a treatable occlusion is to reliably define core infarct size accurately and precisely.

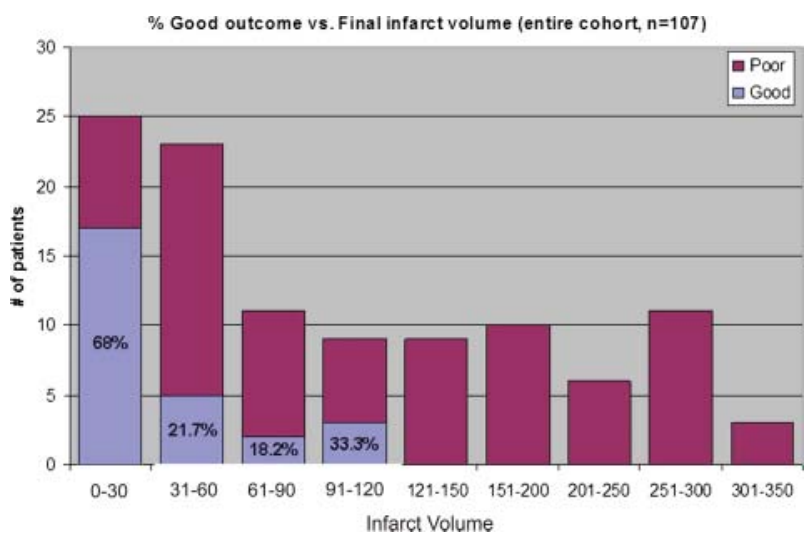

Figure 2 Relationship of final infarct size to clinical outcomes in patients with major anterior circulation occlusions (ACO) treated endovascularly. The bar graphs depict the proportion of good outcomes defined as a modified Rankin Scale score of $0-2$ at 3 months (blue bars) by final infarct volume strata. The data are from 107 patients with $\mathrm{ACO}$. The figure was derived from Yoo et al. ${ }^{4}$
Table 1 Massachusetts General Hospital experience and practice based criteria

\begin{tabular}{ll}
\hline Level & Nearly always valuable in patient care, and successfully obtainable in the \\
1 & vast majority of patients \\
Level & May be valuable in patient care, and is successfully obtainable in most \\
2 & patients \\
Level & Valuable for research purposes and may or may not help in the \\
3 & management of the patient
\end{tabular}

\section{ASSESSMENT OF METHODS National Institutes of Health Stroke Scale}

The NIHSS is a Level 1/Class I test in the assessment of the acute stroke patient. ${ }^{2}$ It helps to quickly determine if the patient is having a stroke and gives an indication of the severity of the stroke. It provides a clinically relevant estimate of the size of the ischemic tissue at risk but cannot differentiate the core from the penumbra.

\section{Non-contrast CT}

NCCT is a Level 1/Class I test for excluding intracranial hemorrhage and mass lesions. ${ }^{2}$ Because of the low sensitivity, NCCT was scored Level 2/Class IIa to detect the infarct core volume during the first few hours after stroke onset. The place of NCCT in the workflow and the order of imaging modalities were also considered and it was agreed that NCCT should be the first test in the evaluation of acute stroke. There are exceptions-for example, MRI could be undertaken as the first imaging study in patients with poor renal function who cannot get CTA, young patients presenting with acute stroke, or patients more likely to have a non-ischemic etiology, such as a mass, seizure, or migraines as the cause of their presenting symptoms.

\section{CT angiography}

CTA is a Level 1/Class I test for the rapid assessment of large vessel occlusion. $^{2}{ }^{13-15}$ It may also be effective for detection of medium and small vessel occlusions, even though they can take longer to find. There was no concern regarding repeatability, reliability, clinical efficacy, or overall utility of CTA. It was agreed that CTA should be performed immediately after the NCCT scan, when feasible.

\section{CT angiography source images}

CTA-SI have been suggested as a surrogate for the infarct core. Some older studies suggested that CTA-SI can image ischemic core. $^{16}{ }^{17}$ However, tissue density in CTA-SI is heavily dependent on the scan timing, cardiac output, the rate of injection, and the osmolality of the contrast media, among other parameters. For these reasons, questions were raised about the

Table 2 Evidence based classification of recommendations

Class I An imaging modality for which there is evidence for and/or general agreement that it is beneficial, useful, and effective in the management of acute stroke

Class II An imaging modality for which there is conflicting evidence and/or divergence of opinion about the usefulness/efficacy in the management of acute stroke

Class Weight of evidence or opinion is in favor of the usefulness or efficacy of lla this imaging modality

Class The usefulness or efficacy is less well established by the evidence or Ilb expert opinion

Class III An imaging test for which there is evidence and/or general agreement that it is not useful or effective, and in some cases may be harmful, in the management of acute stroke patients 
repeatability and reliability of CTA-SI in measuring tissue perfusion. Overall, CTA-SI was judged to be a Level 2/Class III examination in eliciting the core of an ischemic infarct.

\section{Diffusion MRI}

Diffusion MRI is a Level 1/Class I test for the early detection of infarct core. Diffusion weighted imaging (DWI) is nearly $100 \%$ sensitive and specific in diagnosing acute stroke $e^{18-25}$ although it is not perfect in identifying the infarct core. Positron emission tomography (PET) studies have shown that some non-viable tissue occasionally may not demonstrate restricted diffusion ${ }^{26}{ }^{27}$ Also, DWI abnormalities are sometimes reversible. $^{28-33}$ However, reversal of a DWI abnormality is unusual. ${ }^{34} 7$ When DWI reversal does occur, it usually involves only a small part of the lesion. ${ }^{35}$ Also, most of the time, the apparent DWI reversal is actually a pseudo-reversal, in that the tissue involved proceeds to infarction anyway. 7133

\section{Perfusion imaging}

Brain perfusion imaging provides information on cerebral hemodynamics imbedded in parameters such as cerebral blood flow (CBF), cerebral blood volume (CBV), and mean transit time (MTT). Perfusion imaging may provide many types of important information in the care of acute stroke patients. ${ }^{36} 37$ Additionally, much research has been devoted to demonstrating that perfusion imaging can identify the core and penumbra, and that perfusion imaging is useful for identifying patients with major ACOs that are suitable for interventional therapy. 23839 However, evidence based reviews have questioned this. ${ }^{2} 4041$

\section{Perfusion imaging: theoretical considerations}

Perfusion imaging studies the hemodynamic status of the brain at one instant in time, and there is no instantaneous hemodynamic state that uniquely characterizes the infarct core. The hemodynamic response of the brain to ischemia has been characterized with respect to cerebral perfusion pressure, and perfusion parameters are summarized in figure 3 (and in more detail

\begin{tabular}{|c|c|c|c|c|}
\hline & CBV & $\mathrm{CBF}$ & MTT & $T \max$ \\
\hline $\begin{array}{l}\text { Alternate pathway } \\
\text { CPP preserved }\end{array}$ & & & & \\
\hline Compensated low $C$ & & & & \\
\hline Underperfused & & & & \\
\hline $\begin{array}{l}\text { Overperfused } \\
\text { (post-ischemic } \\
\text { hyperperfusion) }\end{array}$ & & & & \\
\hline
\end{tabular}

Figure 3 Changes in hemodynamic parameters that may occur in major anterior circulation occlusions. The change in cerebral perfusion pressure (CPP) may or may not be fully compensated by the collateral circulation. In each of the four scenarios, arrows indicate possible increase or decrease in cerebral blood volume (CBV), cerebral blood flow (CBF), mean transit time (MTT) and Tmax. This is a derivation of the more detailed graph shown in the online supplementary appendix figure $\mathrm{A} 1$. in the online supplementary appendix figure A1). The autoregulatory vasodilation in response to reductions in regional cerebral perfusion pressure would be expected to result in increased, rather than decreased, $\mathrm{CBV}$ in ischemic tissue. Indeed, CBV may be elevated more often than reduced in the infarct core. ${ }^{42}$ More recent proposals have suggested that regions of sufficiently reduced CBF may be labeled as infarct core. This approach has greater pathophysiologic validity in that $\mathrm{CBF}$ reflects the rate of delivery of oxygen and glucose to brain tissue, and reductions in this rate of delivery are responsible for ischemic cell death. However, neither CBF nor any other hemodynamic measurement could ever be used to identify the core. Even complete cessation of blood flow can persist for several minutes without causing irreversible damage. Obviously, perfusion imaging demonstrates no evidence of ongoing perfusion impairment in reperfused tissue.

There are several hemodynamic measurements that are time related with no direct relationship to threats to tissue viability. For example, one of these measurements, Tmax, reflects the time that elapses between arrival of blood in an index artery and its arrival in brain tissue. A delay in the arrival of blood does not directly threaten tissue survival. MTT measures the average amount of time that blood spends in brain tissue, which is usually prolonged in underperfused brain tissue. In the setting of proximal ACO, blood must reach the tissue bed via collateral pathways whose increased circuitry would be expected to result in delayed arrival - that is, increased Tmax. Also, occlusion of a proximal artery would be expected to result in at least some reduction in distal perfusion pressure, which would cause compensatory vasodilation in some of the downstream tissue. This vasodilation by itself should result in prolongation of MTT.

Thus patients with major ACO may or may not have large regions with reduced $\mathrm{CBF}$ but virtually all of these patients will demonstrate large regions of MTT and Tmax elevation. Empirically, we have shown this to be the case, as demonstrated in the online supplementary appendix figure A2. ${ }^{43}{ }^{44}$ In general, the existence of a large region with increased Tmax and MTT may be inferred from the existence of a proximal artery occlusion on CT or MR angiographic imaging alone.

\section{CT perfusion}

Several issues and concerns about CTP were raised in addition to radiation exposure. These concerns-which are related to the workflow, process of obtaining CTP maps, and the utility of the information derived from a CTP study-are summarized below.

\section{CTP process and workflow}

There is a lack of clear guidelines on when CTP should be performed, and how it should be interpreted. There was broad agreement that:

- Quantification of perfusion using CTP is not validated.

- There is high inter-vendor variability.

- There is high intra-vendor variability based on the software version used.

- The variability in CTP maps is as yet unquantified with respect to the variations in heart rate, blood pressure, ejection fraction, rate of infusion, osmolality of IV contrast, rotation time, and temporal resolution of the scanner. ${ }^{45}$

- The efficacy of CTP in improving patient outcomes is unproven.

Studies performed at $\mathrm{MGH}$ directly comparing core volumes by DWI and CTP in patients with severe strokes with ACOs 
revealed statistically significant high correlations between the two methods. However, while there is good correlation in a population, there is a wide clinically relevant discordance between DWI and CTP derived core volumes in individual patients (see online supplementary appendix figure A3).

The above considerations led to the following guidelines on the use of CTP.

- CTP is a Level 3/Class IIb method for early estimation of the infarct core in acute stroke patients. Because CTP is unable to adequately estimate the core, it necessarily follows that it is a Level 3/Class IIb method for estimation of the penumbra.

- CTP has no proven role in selecting ACO patients for IV thrombolysis or endovascular therapy. Its roles should be limited to:

- Research patients

- Patients who cannot get a diffusion weighted MRI

- Perfusion data could be used for other purposes such as hypertensive therapy. However, there are scant data on this application.

\section{MR perfusion}

MRP was deemed preferable to CTP because there is no radiation exposure and it has a generally superior workflow. However, the repeatability, reliability, and clinical efficacy of MRP raise similar concerns to those of CTP, including:

- Quantification using MR perfusion maps is not validated.

- There is high inter-vendor variability.

- The variability of MRP maps with respect to physiologic variables (eg, heart rate, blood pressure, ejection fraction) and scan parameters (eg, rate of infusion, osmolality of IV contrast, rotation time, etc) is unknown.

Carroll and colleagues ${ }^{46}$ performed a Bland-Altman analysis of eight smokers who were imaged with MRP and $\mathrm{H}_{2}{ }^{15} \mathrm{O}$ PET and concluded that "Until reproducibility is improved, $M R$ is not suitable for reliable quantitative perfusion measurements". Other research assessing the reliability of MRP was also reviewed. For example, Takasawa and colleagues ${ }^{47}$ studied perfusion MR (deconvolution method) and PET in five patients, back to back, at a mean time interval of $16 \mathrm{~h}$ after stroke onset. The authors concluded that "MRP appears sufficiently reliable for clinical purposes". However, most participants deliberating on the value of the methods thought that reliability does not override the concerns regarding high variability and low repeatability.

Overall, MRP was judged to be a Level 3/Class IIb technique in the management of acute stroke. There was broad agreement that:

- MRP has no proven role in selecting ACO patients for endovascular therapy. There is preliminary evidence that it may improve patient selection for intravenous thrombolysis but this evidence is currently insufficient to justify MRP's clinical use in this role.

- Clinical indications for MRP may include:

- Research patients

- If perfusion data are deemed essential for evaluating the full clinical picture

- When perfusion data can be used for other purposes such as hypertensive therapy, although there are few data on this.

\section{The clinical penumbra}

The evidence supports that the clinical penumbra, as measured by a combination of the NIHSS and the core determined by
DWI, is the best indicator of a poor outcome in the absence of timely reperfusion. The Prolyse in Acute Cerebral Thromboembolism II trial demonstrated that patients with NIHSS scores $<10$ did not derive a clinical benefit from IAT. ${ }^{48}$ This is due to the relatively good natural history in this subset of patients. ${ }^{49}$ Approximately one-third of the middle cerebral artery M1 segment occlusions present with such low scores. ${ }^{50}$ The evidence best supports the following definition of a clinically significant penumbra: (1) major anterior circulation (ICA terminus or M1) occlusion; (2) NIHSS score $>10$; and (3) small core infarct size (DWI lesion volume $<70$ ). Both NIHSS and DWI are Level 1/Class I tests; together it is judged to be a Level $1 /$ Class I method to assess the clinically relevant penumbra.

\section{THE MGH STROKE IMAGING ALGORITHM}

Based on the conclusions of the evaluation committee on the value of each method delineated above, a new imaging algorithm was proposed and adopted. A diagram of the algorithm is shown in figure 4. Briefly, all patients presenting with a stroke syndrome receive a neurological evaluation, including the NIHSS. NCCT is performed followed by CTA. If NCCT does not demonstrate hemorrhage or large hypodensity, and the patient is within the time window, tPA is prepared while the

\section{MGH Acute Stroke Imaging Algorithm}

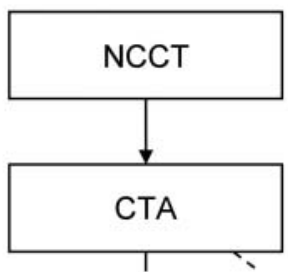

Accessible Proximal Occlusion \& MRI Eligible

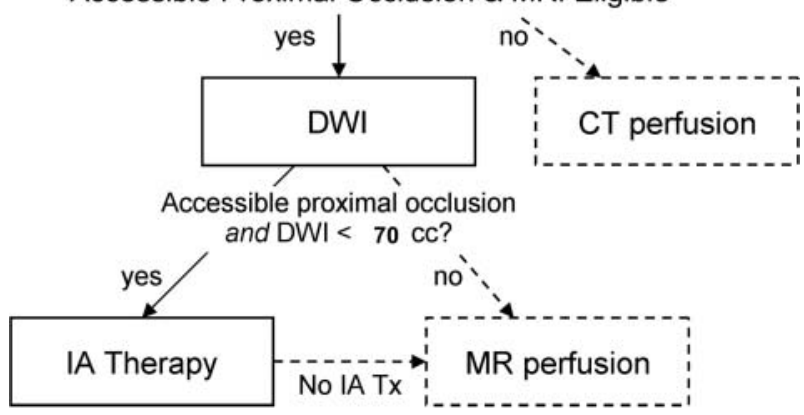

Figure 4 Massachusetts General Hospital acute stroke imaging algorithm for triage of patients with severe ischemic strokes caused by anterior circulation occlusions. All patients undergo non-contrast CT (NCCT) followed by CT angiography (CTA). If the patient has severe neurological deficits (National Institutes of Health Stroke Scale score $\geq 10$ ), no large hypodensity and no hemorrhage on NCCT, and an occlusion is identified of the distal internal carotid artery and/or proximal middle cerebral artery that is accessible by microcatheter, then the patient is immediately evaluated by diffusion MRI if there are no contraindications to MRI. If the diffusion weighted imaging (DWI) lesion is small, defined as $<70 \mathrm{ml}$, then the patient is immediately triaged to endovascular therapy if the patient is otherwise eligible for such treatment. If the patient is not eligible for MRI, he/she may undergo a CT perfusion study for possible guidance for therapy or for prognostic information. Also, if the patient is not eligible for endovascular therapy, CT or MR perfusion may be performed for similar reasons. Only the first step in this algorithm (NCCT) and the time from stroke onset are needed for the decision to treat with intravenous tissue plasminogen activator. IA, intra-arterial. 
CTA is performed, and infusion begins once it is prepared. If the patient has a distal ICA and/or proximal middle cerebral artery occlusion, he/she is moved to the MRI scanner where diffusion $\mathrm{MRI}$ is performed. If the DWI lesion is small $(<70 \mathrm{ml})$, the patient is sent for IAT if the additional clinical and medical criteria are met. Perfusion imaging with CT or MRI may be performed if these conditions are not met, the patient cannot be scanned by MRI, or is not otherwise eligible for IAT and there is relevant clinical information that may be provided by the perfusion data.

After the algorithm was adopted, there was a significant decline in the number of perfusion CT examinations performed, as shown in the online supplementary appendix figure A4. From 40-50 CTP examinations per month in stroke patients performed during the peak years of 2005-2008, it fell to approximately 10 per month. There has been no discernible effect on patient outcomes.

Contributors All authors contributed to the manuscript and study.

Competing interests JMR is on the imaging committee of the DIAS trial for Lundbeck Pharmaceuticals. MHL has research support from GE Healthcare and is a consultant for Millenium Pharmaceuticals.

Provenance and peer review Commissioned; internally peer reviewed.

Open Access This is an Open Access article distributed in accordance with the Creative Commons Attribution Non Commercial (CC BY-NC 3.0) license, which permits others to distribute, remix, adapt, build upon this work non-commercially, and license their derivative works on different terms, provided the original work is properly cited and the use is non-commercial. See: http://creativecommons.org/ licenses/by-nc/3.0/

\section{REFERENCES}

1. Baron JC. Mapping the ischaemic penumbra with PET: implications for acute stroke treatment. Cerebrovasc Dis 1999;9:193-201.

2. Jauch EC, Saver JL, Adams HP Jr, et al. Guidelines for the early management of patients with acute ischemic stroke: a guideline for healthcare professionals from the American Heart Association/American Stroke Association. Stroke 2013;44:870-947.

3. Smith WS, Lev MH, English JD, et al. Significance of large vessel intracranial occlusion causing acute ischemic stroke and TIA. Stroke 2009;40:3834-40.

4. Yoo AJ, Chaudhry ZA, Nogueira RG, et al. Infarct volume is a pivotal biomarker after intra-arterial stroke therapy. Stroke 2012;43:1323-30.

5. Zaidi SF, Aghaebrahim A, Urra X, et al. Final infarct volume is a stronger predictor of outcome than recanalization in patients with proximal middle cerebral artery occlusion treated with endovascular therapy. Stroke 2012;43:3238-44.

6. Yoo AJ, Verduzco LA, Schaefer PW, et al. MRI-based selection for intra-arterial stroke therapy: value of pretreatment diffusion-weighted imaging lesion volume in selecting patients with acute stroke who will benefit from early recanalization. Stroke 2009; 40:2046-54.

7. Lansberg MG, Straka M, Kemp S, et al. MRI profile and response to endovascular reperfusion after stroke (DEFUSE 2): a prospective cohort study. Lancet Neurol 2012:11:860-7.

8. Lansberg MG, Thijs VN, Bammer R, et al. Risk factors of symptomatic intracerebral hemorrhage after tPA therapy for acute stroke. Stroke 2007;38:2275-8.

9. Singer $\mathbf{O C}$, Humpich MC, Fiehler J, et al. Risk for symptomatic intracerebral hemorrhage after thrombolysis assessed by diffusion-weighted magnetic resonance imaging. Ann Neurol 2008;63:52-60.

10. Jovin TG, Yonas $\mathrm{H}$, Gebel JM, et al. The cortical ischemic core and not the consistently present penumbra is a determinant of clinical outcome in acute middle cerebral artery occlusion. Stroke 2003;34:2426-33.

11. Hill MD, Rowley HA, Adler F, et al. Selection of acute ischemic stroke patients for intra-arterial thrombolysis with pro-urokinase by using ASPECTS. Stroke 2003:34:1925-31.

12. Hill MD, Demchuk AM, Tomsick TA, et al. Using the baseline CT scan to select acute stroke patients for IV-IA therapy. AJNR Am J Neuroradiol 2006;27:1612-16.

13. Torres-Mozqueda $\mathbf{F}$, He J, Yeh IB, et al. An acute ischemic stroke classification instrument that includes CT or MR angiography: the Boston Acute Stroke Imaging Scale. AJNR Am J Neuroradiol 2008;29:1111-17.

14. Cipriano LE, Steinberg ML, Gazelle GS, et al. Comparing and predicting the costs and outcomes of patients with major and minor stroke using the Boston Acute Stroke Imaging Scale neuroimaging classification system. AJNR Am J Neuroradiol 2009:30:703-9.
15. Gonzalez RG, Lev MH, Goldmacher GV, et al. Improved outcome prediction using CT angiography in addition to standard ischemic stroke assessment: results from the STOPStroke study. PLoS One 2012;7:e30352.

16. Hunter GJ, Hamberg LM, Ponzo JA, et al. Assessment of cerebral perfusion and arterial anatomy in hyperacute stroke with three-dimensional functional CT: early clinical results. AJNR Am J Neuroradiol 1998;19:29-37.

17. Pulli B, Schaefer PW, Hakimelahi R, et al. Acute ischemic stroke: infarct core estimation on CT angiography source images depends on CT angiography protocol. Radiology 2012;262:593-604.

18. Lövblad KO, Laubach $\mathrm{HJ}$, Baird AE, et al. Clinical experience with diffusion-weighted MR in patients with acute stroke. AJNR Am J Neuroradiol 1998:19:1061-6.

19. Singer MB, Chong J, Lu D, et al. Diffusion-weighted MRI in acute subcortical infarction. Stroke 1998;29:133-6.

20. Gonzalez RG, Schaefer PW, Buonanno FS, et al. Diffusion-weighted MR imaging: diagnostic accuracy in patients imaged within 6 hours of stroke symptom onset. Radiology 1999;210:155-62.

21. Urbach H, Flacke $\mathrm{S}$, Keller $\mathrm{E}$, et al. Detectability and detection rate of acute cerebral hemisphere infarcts on CT and diffusion-weighted MRI. Neuroradiology 2000;42:722-7

22. van Everdingen KJ, van der Grond J, Kappelle LJ, et al. Diffusion-weighted magnetic resonance imaging in acute stroke. Stroke 1998;29:1783-90.

23. Perkins CJ, Kahya E, Roque CT, et al. Fluid-attenuated inversion recovery and diffusion- and perfusion-weighted MRI abnormalities in 117 consecutive patients with stroke symptoms. Stroke 2001;32:2774-81.

24. Fiebach JB, Schellinger PD, Jansen 0, et al. CT and diffusion-weighted MR imaging in randomized order: diffusion-weighted imaging results in higher accuracy and lower interrater variability in the diagnosis of hyperacute ischemic stroke. Stroke 2002;33:2206-10.

25. Mullins ME, Schaefer PW, Sorensen AG, et al. CT and conventional and diffusion-weighted MR imaging in acute stroke: study in 691 patients at presentation to the emergency department. Radiology 2002;224:353-60.

26. Shimosegawa $\mathbf{E}$, Hatazawa J, Ibaraki $\mathrm{M}$, et al. Metabolic penumbra of acute brain infarction: a correlation with infarct growth. Ann Neurol 2005;57:495-504.

27. Guadagno JV, Warburton EA, Jones PS, et al. The diffusion-weighted lesion in acute stroke: heterogeneous patterns of flow/metabolism uncoupling as assessed by quantitative positron emission tomography. Cerebrovasc Dis 2005;19:239-46.

28. Kidwell CS, Saver JL, Mattiello J, et al. Thrombolytic reversal of acute human cerebral ischemic injury shown by diffusion/perfusion magnetic resonance imaging. Ann Neurol 2000;47:462-9.

29. Neumann-Haefelin T, Wittsack HJ, Wenserski F, et al. Diffusion- and perfusion-weighted MRI in a patient with a prolonged reversible ischaemic neurological deficit. Neuroradiology 2000:42:444-7.

30. Fiehler J, Foth M, Kucinski T, et al. Severe ADC decreases do not predict irreversible tissue damage in humans. Stroke 2002;33:79-86.

31. Kidwell CS, Saver JL, Starkman S, et al. Late secondary ischemic injury in patients receiving intraarterial thrombolysis. Ann Neurol 2002;52:698-703.

32. Oppenheim C, Lamy C, Touze $E$, et al. Do transient ischemic attacks with diffusion-weighted imaging abnormalities correspond to brain infarctions? AJNR Am J Neuroradiol 2006;27:1782-7.

33. Campbell BC, Purushotham A, Christensen S, et al. The infarct core is well represented by the acute diffusion lesion: sustained reversal is infrequent. $J$ Cereb Blood Flow Metab 2012;32:50-6.

34. Grant PE, He J, Halpern EF, et al. Frequency and clinical context of decreased apparent diffusion coefficient reversal in the human brain. Radiology 2001:221:43-50.

35. Parsons MW, Yang 2, Barber PA, et al. Perfusion magnetic resonance imaging maps in hyperacute stroke: relative cerebral blood flow most accurately identifies tissue destined to infarct. Stroke 2001;32:1581-7.

36. Lev MH. Perfusion imaging of acute stroke: its role in current and future clinical practice. Radiology 2013;266:22-7.

37. Zhu G, Michel P, Aghaebrahim A, et al. Computed tomography workup of patients suspected of acute ischemic stroke: perfusion computed tomography adds value compared with clinical evaluation, noncontrast computed tomography, and computed tomography angiogram in terms of predicting outcome. Stroke Published Online First: 12 February 2013. doi: 10.1161/STROKEAHA.111.674705

38. Wintermark M, Albers GW, Alexandrov AV, et al. Acute stroke imaging research roadmap. Stroke 2008;39:1621-28.

39. Wintermark M, Flanders AE, Velthuis B, et al. Perfusion-CT assessment of infarct core and penumbra: receiver operating characteristic curve analysis in 130 patients suspected of acute hemispheric stroke. Stroke 2006;37:979-85.

40. Schellinger PD, Bryan RN, Caplan LR, et al. Evidence-based guideline: the role of diffusion and perfusion MRI for the diagnosis of acute ischemic stroke: report of the Therapeutics and Technology Assessment Subcommittee of the American Academy of Neurology. Neurology 2010;75:177-85.

41. Gonzalez RG. Low signal, high noise and large uncertainty make CT perfusion unsuitable for acute ischemic stroke patient selection for endovascular therapy. $J$ Neurointervent Surg 2012;4:242-5. 
42. Deipolyi AR, Wu O, Macklin EA, et al. Reliability of cerebral blood volume maps as a substitute for diffusion-weighted imaging in acute ischemic stroke. J Magn Reson Imaging 2012;36:1083-7.

43. Copen WA, Rezai Gharai L, Barak ER, et al. Existence of the diffusion-perfusion mismatch within 24 hours after onset of acute stroke: dependence on proximal arterial occlusion. Radiology 2009;250:878-86.

44. Hakimelahi R, Yoo AJ, He J, et al. Rapid identification of a major diffusion/ perfusion mismatch in distal internal carotid artery or middle cerebral artery ischemic stroke. BMC Neurol 2012;12:132.

45. Fiorella D, Heiserman J, Prenger $\mathrm{E}$, et al. Assessment of the reproducibility of postprocessing dynamic CT perfusion data. AJNR Am J Neuroradiol 2004;25:97-107.

46. Carroll TJ, Teneggi $\mathrm{V}$, Jobin $\mathrm{M}$, et al. Absolute quantification of cerebral blood flow with magnetic resonance, reproducibility of the method, and comparison with $\mathrm{H} 2$
(15)0 positron emission tomography. J Cereb Blood Flow Metab 2002;22: 1149-56.

47. Takasawa M, Jones PS, Guadagno JV, et al. How reliable is perfusion MR in acute stroke? Validation and determination of the penumbra threshold against quantitative PET. Stroke 2008;39:870-7.

48. Furlan A, Higashida R, Wechsler L, et al. Intra-arterial prourokinase for acute ischemic stroke. The PROACT II study: a randomized controlled trial. Prolyse in Acute Cerebral Thromboembolism. JAMA 1999;282:2003-11.

49. Yoo AJ, Barak ER, Copen WA, et al. Combining acute diffusion-weighted imaging and mean transmit time lesion volumes with National Institutes of Health Stroke Scale Score improves the prediction of acute stroke outcome. Stroke 2010;41:1728-35.

50. Maas MB, Furie KL, Lev MH, et al. National Institutes of Health Stroke Scale score is poorly predictive of proximal occlusion in acute cerebral ischemia. Stroke 2009; 40:2988-93. 